\title{
Kin discrimination and outer membrane exchange in Myxococcus xanthus: A comparative analysis among natural isolates
}

\author{
Sébastien Wielgoss ${ }^{1}$ (D) | Francesca Fiegna ${ }^{1}$ | Olaya Rendueles ${ }^{1,2} \mid$ Yuen-Tsu N. Yu $^{1}$ | \\ Gregory J. Velicer ${ }^{1}$
}

${ }^{1}$ Institute of Integrative Biology, ETH Zürich, Zürich, Switzerland

${ }^{2}$ Microbial Evolutionary Genomics Unit, Institut Pasteur, Paris, France

Correspondence

Sébastien Wielgoss and Gregory J. Velicer, Institute of Integrative Biology, ETH Zürich, Universitätstrasse 16, 8092 Zürich, Switzerland

Emails: sebastien.wielgoss@env.ethz.ch (SW) and gregory.velicer@env.ethz.ch (GJV)

Funding information European Molecular Biology Organization; FP7 People: Marie-Curie Actions, Grant/ Award Number: FP7-PEOPLE-2012-IEF331824

\begin{abstract}
Genetically similar cells of the soil bacterium Myxococcus xanthus cooperate at multiple social behaviours, including motility and multicellular development. Another social interaction in this species is outer membrane exchange (OME), a behaviour of unknown primary benefit in which cells displaying closely related variants of the outer membrane protein TraA transiently fuse and exchange membrane contents. Functionally incompatible TraA variants do not mediate OME, which led to the proposal that TraA incompatibilities determine patterns of intercellular cooperation in nature, but how this might occur remains unclear. Using natural isolates from a centimetre-scale patch of soil, we analyse patterns of TraA diversity and ask whether relatedness at TraA is causally related to patterns of kin discrimination in the form of both colony-merger incompatibilities (CMIs) and interstrain antagonisms. A large proportion of TraA functional diversity documented among global isolates is predicted to be contained within this $\mathrm{cm}$-scale population. We find evidence of balancing selection on the highly variable PA14-portion of TraA and extensive transfer of traA alleles across genomic backgrounds. CMls are shown to be common among strains identical at TraA, suggesting that CMIs are not generally caused by TraA dissimilarity. Finally, it has been proposed that interstrain antagonisms might be caused by OME-mediated toxin transfer. However, we predict that most strain pairs previously shown to exhibit strong antagonisms are incapable of OME due to TraA dissimilarity. Overall, our results suggest that most documented patterns of kin discrimination in a natural population of $M$. xanthus are not causally related to the TraA sequences of interactants.
\end{abstract}

KEYWORDS

behaviour, colony-merger incompatibility, myxobacteria, outer membrane exchange, phylogeny, sociobiogeography

\section{1 | INTRODUCTION}

Microbes exhibit various forms of kin discrimination, in which social behaviours and their effects vary as a function of genetic relatedness between interactants at one or more loci (Strassmann, Gilbert, \&
Queller, 2011). A major form of microbial kin discrimination is colony-merger incompatibility (CMI; Dienes, 1946; Stefanic, Kraigher, Lyons, Kolter, \& Mandic-Mulec, 2015; Velicer \& Vos, 2009). In CMI, genetically distinct colonies that meet due to motility exhibit altered behaviour at their colony interface relative to encounters between 
colonies of the same genotype (Figure 1). CMls have been documented among natural isolates in several species of swarming bacteria, including Proteus mirabilis (Budding, Ingham, Bitter, Vandenbroucke-Grauls, \& Schneeberger, 2009), Myxococcus xanthus (Vos \& Velicer, 2009) and Bacillus subtilis (Stefanic et al., 2015), and have been shown to evolve pervasively among experimental lineages of M. xanthus (Rendueles, Zee et al., 2015).

Myxococcus xanthus engages in multiple cooperative behaviours, including coordinated motility and development of multicellular fruiting bodies (Hodgkin \& Kaiser, 1979; Kaiser \& Welch, 2004; Wu \& Kaiser, 1995). CMls can strongly alter patterns of cooperation in Myxococcus and can alter the composition of social groups when motile colonies meet in structured habitats (Kraemer, Wielgoss, Fiegna, \& Velicer, 2016; Rendueles, Amherd, \& Velicer, 2015; Rendueles, Zee et al., 2015; Vos \& Velicer, 2009). In particular, CMls between $M$. xanthus genotypes isolated from a local patch of soil can greatly reduce mixing of distinct genotypes within individual fruiting bodies that form at colony interfaces (Rendueles, Amherd et al., 2015). In contrast, control strains that do not exhibit CMI readily coaggregate and generate high frequencies of chimeric fruiting bodies where colonies meet.

Natural M. xanthus populations are highly structured, as genetic diversity generally increases with distance between sampling points from micrometre to global scales (Kraemer \& Velicer, 2011; Kraemer et al., 2016; Vos \& Velicer, 2008). Fruiting bodies emerging from soil samples are frequently internally diverse, but such within-group diversity tends to be endemic and low relative to diversity across even neighbouring fruiting bodies or larger spatial scales (Kraemer \& Velicer, 2011; Kraemer et al., 2016). Distinct genotypes coresiding in local patches of soil at millimetre-centimetre scales often exhibit strong CMls when motile colonies meet (Kraemer et al., 2016; Vos \& Velicer, 2009), and also commonly display strong antagonisms when cells are mixed into chimeric groups during development (Rendueles, Amherd et al., 2015; Vos \& Velicer, 2009) or growth (Rendueles, Amherd et al., 2015).

Myxococcus xanthus exhibits an intriguing type of cell-cell interaction known as outer membrane exchange (OME; Nudleman, Wall, \& Kaiser, 2005; Pathak et al., 2012; Wei, Pathak, \& Wall, 2011). During OME, motile cells on solid surfaces transiently fuse and exchange outer membrane components, including lipids and lipoproteins (Ducret, Fleuchot, Bergam, \& Mignot, 2013; Nudleman et al., 2005; Pathak et al., 2012). Such exchange of cellular resources by OME could play a major role in M. xanthus sociality (Bretl \& Kirby, 2016; Pathak, Wei, Dey, \& Wall, 2013; Pathak et al., 2012; Vassallo et al., 2015). Importantly, however, the primary benefits of OME in nature remain unknown. Loss-of-function mutations for OME have not been
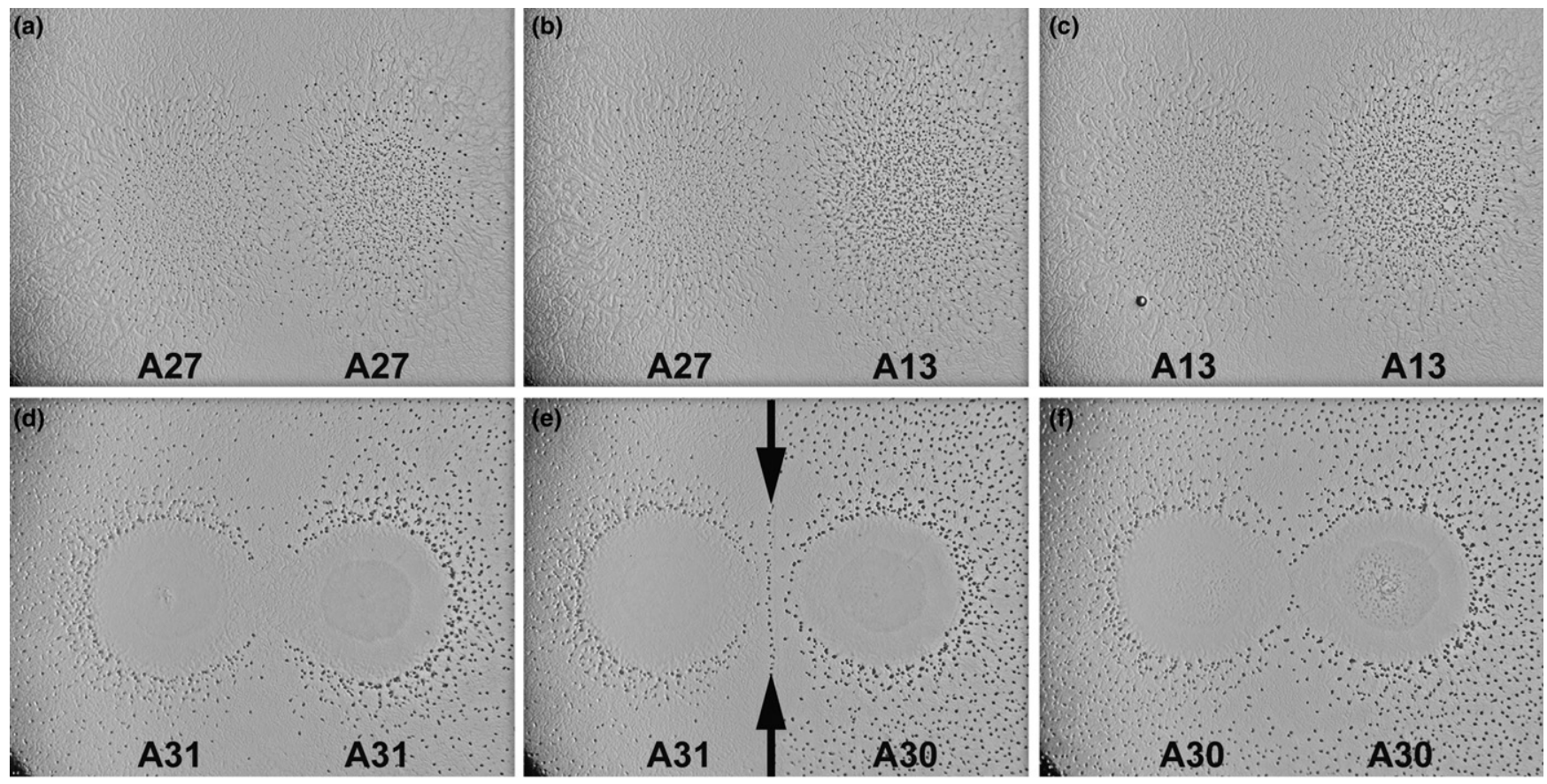

$0.5 \mathrm{~cm}$

FIGURE 1 Examples of colony-interface phenotypes for compatible (a-c) and incompatible (d-f) strain pairs. Panels (a), (c), (d) and (f) depict control self-self encounters for four different strains (A27, A13, A31 and A30, respectively) in which two colonies of the same isolate meet on a low-nutrient agar plate after having been inoculated at separate points $(1 \mathrm{~cm}$ apart). Dark spots depict fruiting bodies. Panel (b) shows a colony encounter between distinct strains (A27 and A13) in which the population-level swarming and fruiting-body formation patterns of each strain are not visually distinguishable from self-self controls. In contrast, in panel (e) the colony-interface phenotype for A31 (left) and A30 (right) differs from the interfaces in both respective self-self controls and thus exhibits a colony-merger incompatibility. In particular, a line of fruiting bodies (denoted by two arrows) forms at the interface of A31-A30 colonies that does not form at either of the self-self interfaces. The scale bar on the bottom left is representative for all images 
found to adversely affect other social traits such as motility and spore production (Pathak et al., 2012). Nonetheless, the gene traA, which is required for $\mathrm{OME}$, is present in a range of myxobacterial species and M. xanthus strains (Pathak et al., 2012), suggesting that some function of this gene, including possibly OME, is beneficial.

The TraA protein is predicted to be a membrane receptor and adhesin (Pathak et al., 2012) that mediates OME in conjunction with TraB (Cao \& Wall, 2017). TraA contains four major structural features including a PA14-like domain (Pathak et al., 2012) that is found in a wide variety of prokaryotic and eukaryotic proteins that include adhesins, amidases, glycosidases, glycosyltransferases, proteases and toxins (Collier \& Young, 2003; Goossens \& Willaert, 2010; Oohata, Nakagawa, Tasaka, \& Fujii, 1997; Rigden, Mello, \& Galperin, 2004; Taylor \& Drickamer, 2014; Zupancic et al., 2008). The PA14 domain of TraA is highly polymorphic and PA14 sequences of interacting cells must be highly similar for OME to occur (Pathak et al., 2013). Tests for OME across a broad range of $M$. xanthus isolates have revealed a modular OME network structure in which TraA has diversified into a small number of OME compatibility categories (Pathak et al., 2013). While this phylogenetic structure was highly correlated with overall variation in the PA14 domain, differences at a single amino acid position (205) can readily generate OME incompatibilities between otherwise isogenic strains (Cao \& Wall, 2017).

The traA gene has been labelled a "greenbeard" locus that allows the exchange of cooperative benefits of OME between cells with the same TraA functional type and prevents such exchanges between cells dissimilar at TraA (e.g., see Cao, Dey, Vassallo, \& Wall, 2015; Cao \& Wall, 2017; Vassallo \& Wall, 2016; Vassallo et al., 2015; Wall, 2014, 2016). However, there remain at least two challenges to evaluating this hypothesis. First, as noted above, the primary benefits of OME for either donors or receivers in nature remain unclear. A hypothetical selective benefit labelled "outer membrane homeostasis" has been proposed (Vassallo \& Wall, 2016; Vassallo et al., 2015) but, in our view, not conclusively tested. Second, OME-related assays of cells with nonmatching TraA variants have involved forced mixing of cells rather than encounters between distinct colonies caused by motility (e.g., Cao \& Wall, 2017; Pathak et al., 2013). This is relevant in the light of the intense spatial structure of M. xanthus kin groups in nature, where incompatible TraA variants are likely to make physical contact with one another, if ever, at colony-colony interfaces. This consideration prompts several questions. What happens when motile colonies of natural isolates that display matching or nonmatching TraA variants meet? Do the phenotypes of such colony interfaces (e.g., CMls) correlate with TraA identity in any manner? If any such correlation exists, is there any evidence that TraA-OME identity is the causal driver of the correlation as opposed to being a secondary, noncausal correlate?

This study has several goals subsumed under a broader aim of ultimately understanding the potential role of TraA in M.xanthus natural social interactions, particularly with respect to the greenbeard hypothesis. To this end, here we use comparative analysis of M. xanthus natural isolates, including 78 isolates from a focal centimetre-scale population from Tübingen, Germany, to examine traA/
TraA diversity at both fine and broad spatial scales. We compare the traA phylogeny of $\mathrm{cm}$-scale isolates to that of globally distributed isolates with respect to both phylogenetic structure and patterns of predicted functional TraA identity and we test for evidence of selection on traA. We further assess the degree to which patterns of relatedness at traA correlate with signals of broader genomic relatedness. Finally, we test two ways that TraA identity (with respect to OME functionality) might causally affect patterns of cooperation and conflict in natural populations.

First, functional divergence between TraA allotypes that might make contact at colony interfaces could causally contribute to CMIs. Because TraA is a predicted adhesin, contact between colonies with nonmatching TraA allotypes might induce different behavioural responses than do encounters between cells of the same TraA functional type (or between cells that differ in whether they produce any functional TraA variant at all). If TraA incompatibilities are a primary determinant of CMls in natural populations, CMls should be both common between genotypes bearing functionally nonmatching TraA allotypes and rare between strains with matching TraA proteins. If CMls are common between strains sharing the same TraA type, then variation at other loci must often be responsible. A recent study showing that TraA-independent CMls can rapidly evolve in laboratory populations (Rendueles, Zee et al., 2015) suggests that CMls among natural isolates may also be largely independent of TraA identity, but this remains untested. Any effects of TraA incompatibility on colony encounters might be mediated solely by whether or not OME occurs between the relevant cells but might also occur independently of $\mathrm{OME}$, for example if contact between highly divergent TraA allotypes directly influences motility behaviour in a manner not mediated by OME.

Second, and inversely from the greenbeard hypothesis, a recent discovery suggests that TraA similarity may in fact often allow OMEspecific antagonisms that would not occur between strains incapable of engaging in OME together. Specifically, killing of closely related M. xanthus strains can occur due to OME and the presence of the polyploid prophage Mx alpha (Dey et al., 2016; Vassallo et al., 2017; Wielgoss et al., 2016). This antagonism is mediated by prophageencoded toxins delivered by OME from toxin-resistant to toxin-sensitive strains (Dey et al., 2016). Wielgoss et al. (2016) thus proposed that OME-mediated toxins might contribute to natural patterns of antagonism in local natural populations. If most strong antagonisms in natural populations are mediated by OME, then such antagonisms should occur predominantly between strains with functionally compatible TraA sequences. Here, we test whether pairs of $\mathrm{cm}$-scale Tübingen isolates that exhibit previously documented antagonisms are predicted to be compatible for OME based on their degree of similarity at the PA14-region of TraA.

\section{2 | MATERIALS AND METHODS}

\section{1 | Bacterial strains}

Colony-interface phenotype assays were performed with (a) 14 of the 17 M. xanthus isolates examined in Pathak et al. (2013) and with 
(b) a large subset $(n=51)$ of the 78 isolates isolated from a centimetre-scale patch of soil in Tübingen, Germany, reported by Vos and Velicer (2006). Strains beginning with "A" are from the focal cmscale Tübingen population. One assay was performed with the laboratory reference strain GJV1, which is a recent derivative of the standard laboratory strain DK1622 (Velicer et al., 2006), and ultimately derived from strain DK101. Both DK1622 and GJV1 lack an 222-kb genomic region originally present in DK101 (Chen, Kuspa, Keseler, \& Shimkets, 1991; Dey et al., 2016). The region in question is highly variable and contains multiple copies of prophage element Mx alpha, which encodes a recently discovered bacteriocin, sitA (Dey et al., 2016; Vassallo et al., 2017; Wielgoss et al., 2016). The remaining strains are other natural isolates from globally distributed origins. Strain DK897 was renamed GVK897 by Pathak et al. (2013), but here we continue to use its original name as used in Fiegna and Velicer (2005), Vos and Velicer (2009) and Rajagopalan, Wielgoss, Lippert, Velicer, and Kroos (2015).

\subsection{Phylogenetic inference}

We sequenced the entire coding sequence of traA (GenBank Accession nos. $\mathrm{MH} 251641-\mathrm{MH} 251718$ ) from all 78 natural isolates reported by Vos and Velicer (2006) and a recently identified outgroup for M. xanthus, MC359c15 (Rajagopalan et al., 2015). Primer sequences are listed in the Supporting material and methods (Supporting Information Appendix S1). Nucleotide sequences for traA were globally aligned using Muscle by accounting for codons in MEGA version 6.06 (Tamura, Stecher, Peterson, Filipski, \& Kumar, 2013). This alignment was then phylogenetically analysed in two different ways: first for higher phylogenetic resolution, we inferred the phylogenetic relationships for the aligned nucleotide sequences using maximum likelihood assuming the appropriate model of sequence evolution, $H K Y+G+I$ (with $G$, the gamma shape parameter, set at 0.49 , and I, the fraction of invariant sites, set at 0.47 ), and assessed statistical support of each branch in the tree based on bootstrapping by running 1,000 pseudoreplicates in MEGA version 6.06. Second, we followed the same Bayesian phylogenetic inference protocol described in Pathak et al. (2013) for their previously published traA sequences from global isolates, including an identical alignment (GenBank Accession nos.: JX876748-JX876762), and reconfirmed the published tree topology with virtually identical posterior likelihood values. Third, we combined the amino acid sequence of PA14 from Pathak et al. (2013) with our own (translated) sequence data set for $\mathrm{cm}$-scale isolates and inferred the joint phylogeny after performing a global alignment with Muscle accounting for codons in MEGA version 6.06 (Tamura et al., 2013), which was then modified manually to follow the exact same alignment as published by Cao and Wall (2017) for cross-comparison. In particular, we conducted both a maximumlikelihood (ML) tree inference in MEGA version 6.06 and a Bayesian tree inference in MrBayes (Ronquist et al., 2012), both times assuming the Dayhoff amino acid model with frequencies $(+F)$ of sequence evolution and gamma distributed rates among sites with invariant positions $(+\mathrm{G}+\mathrm{l}$; six gamma categories) as suggested using ProtTest3
(Darriba, Taboada, Doallo, \& Posada, 2011). For the ML tree inference, we ran 1,000 bootstrap pseudoreplicates, and for the Bayesian inference, two parallel trees were run for 2,600,000 generations with an initial burn-in period of $25 \%$ of the total run, and a total of 7,812 trees sampled, until both the standard deviation of split frequencies settled below 0.007 and the potential scale reduction factors FOR THE tree length approached 1.0. The latter signifies that the tree searches were run for a sufficient number of generations (Gelman \& Rubin, 1992). Finally, to visualize the presence of recombination (see below), we contrasted the phylogenetic trees for both the aligned traA nucleotide sequences of five $\mathrm{cm}$-scale and six global isolates with an aligned four-gene concatemer (i.e., a multilocus sequence tag, MLST) consisting of $\operatorname{csg} A$, fibA, sgIK and $r p o D$ representing their genetic backgrounds. The latter represented the genetic backgrounds of several natural isolates that (a) were studied by Pathak et al. (2013) for OME phenotypes and (b) for which MLST data were available in public databases (referenced in Vos \& Velicer, 2006, 2009). The phylogenies were inferred with maximum likelihood in MEGA version 6.06 assuming the appropriate model for sequence evolution (MLST: T3P +G +l; traA: HKY +G). Statistical support of each split in both trees was assessed based on bootstrapping by running 1,000 pseudoreplicates.

\section{3 | Tests for recombination and selective neutrality}

Prior to performing neutrality tests, we inspected the set of 18 unique traA sequences for the presence of recombination by applying the GARD method (Pond, Posada, Gravenor, Woelk, \& Frost, 2006) as implemented in the HyPhy package (Pond, Frost, \& Muse, 2005) on the Datamonkey server (Delport, Poon, Frost, \& Pond, 2010; Pond \& Frost, 2005). We statistically tested the assumption that variation in traA is neutral using both Tajima's $D$ (Tajima, 1989) and Fu \& Li's F (Fu \& Li, 1993) tests. The latter is one of the most powerful neutrality tests but more sensitive to recombination (RamirezSoriano, Ramos-Onsins, Rozas, Calafell, \& Navarro, 2008), while the former is less powerful, but less sensitive to recombination (RamirezSoriano et al., 2008; Schierup \& Hein, 2000). More specifically, we tested neutrality of mutations in the entire coding sequence of traA as well as independently for its major subregions, that is, the highly variable PA14-like and the cysteine-rich repeat regions. These tests were performed with the web-based version of neutralitytest.c (http://wwwabi.snv.jussieu.fr/achaz/neutralitytest.html) as implemented by Guillaume Achaz.

\subsection{Rarefaction curves of allelic richness at TraA}

To highlight natural diversity at TraA-PA14-domains, we inferred rarefaction curves of allelic richness up to $n=78$ (our entire sequence set) using a custom $R$ script. Of the various functions tested for curve fitting using $R$ base function nls with appropriate starting values, two visually close fits were subsequently tested statistically, namely: 
$f(x)=a x^{b}+c$, the power law function, and,

$$
f(x)=a \ln x+b, \text { the logarithmic function. }
$$

Using the Akaike information criterion (AIC), the power law function was a better fit $(\mathrm{AIC}=-39.4)$ than the logarithmic function $(\mathrm{AIC}=53.0)$.

\subsection{Assessing the correlation between TraA and MLST relatedness}

We tested how pairwise traA genetic distances are correlated with genomic relatedness, the latter being represented by MLST as published in Vos and Velicer (2009). For this, we inferred Spearman's $r$ and performed a Mantel test (Mantel, 1967) with 10,000 replicates as implemented in the ade4-package (Chessel, Dufour, \& Thioulouse, 2004). We further binned each strain's traA allele by corresponding MLST, and inferred average genetic diversity, $\pi$, within vs. across MLST groups as implemented in MEGA version 6.06. Finally, we calculated haplotype diversities for each MLST group, defined as

$$
H=1-\sum_{i=1}^{n} p_{i}^{2},
$$

where $i$ is the index of the ith MLST bin of a total of $n=14$ observed TraA-PA14 domains.

\section{6 | Colony-interface phenotype assay}

To test whether relatedness at traA predicts social compatibility, we compared a total of 77 colony-interface phenotypes between colonies of distinct isolates relative to those of self-self controls (Kraemer et al., 2016; Rendueles, Zee et al., 2015). A set of 51 of the 78 natural isolates were inoculated on CTT (Bretscher \& Kaiser, 1978) 1.5\%-agar plates and incubated for $4-5$ days at $32^{\circ} \mathrm{C}, 90 \% \mathrm{rH}$. Each strain was transferred into eight $\mathrm{ml}$ of CTT liquid and grown for approximately $24 \mathrm{hr}$ at $32^{\circ} \mathrm{C}$, at $300 \mathrm{rpm}$ in an orbital shaker. Liquid cultures at midexponential phase were centrifuged $(4,500 \mathrm{~g}, 15 \mathrm{~min})$ and resuspended in TPM buffer to $\sim 510^{9}$ cells/ml. Notably, OME occurs on hard $(>1 \% \mathrm{~m} / \mathrm{v})$ agar surfaces and during swarming motility (e.g., Pathak et al., 2012, 2013). We initiated each assay by inoculating two $10-\mu \mathrm{l}$ spots on a reduced, $0.3 \%$, casitone CTT $1.5 \%$-agar plate at a distance of $1 \mathrm{~cm}$. Thus, each plate contained either two spots of the same isolate (self-self controls) or one spot each of two independent isolates. After incubation for 6 days at $32^{\circ} \mathrm{C}, 90 \% \mathrm{rH}$, the interfaces between distinct-isolate colonies were compared to those of self-self controls (see Figure 1a,c,d,f for example), and the colony-interface phenotypes were scored as being either visually indistinguishable from those of self-self controls for both isolates or visually distinct from the self-self control for at least one of the two isolates. Paired isolates with colony-interface phenotypes that were indistinguishable from controls for all assay replicates were categorized as "compatible" (see Figure $1 \mathrm{~b}$ for an example), whereas paired isolates with phenotypes that were clearly distinguishable from controls for all assay replicates were categorized as "incompatible" (see Figure 1e for an example).
Pairs exhibiting inconsistent interaction phenotypes across replicates were categorized as "variable." Each isolate pairing and parallel controls were replicated at least three times.

\subsection{Disruption of traA in GJV1}

An internal fragment ( 780 bp) of the traA gene of GJV1 was amplified from genomic DNA using NEB's Phusion ${ }^{\circledR}$ High Fidelity DNA polymerase with primers GV751 (TCACTGTCTTGTCGGTGTGCCT, positions 68-89 of traA, which has 2,160 nucleotides (Pathak et al., 2012) and GV753 (CGTCCGCCCTCCGTGTGGAAGAA, positions 832-854 of traA). The resulting amplicon was cloned into Invitrogen's $p C R B$ lunt ${ }^{\circledR}$-vector to create plasmid pTraA. A traA knock-in mutant was constructed by transforming the kanamycin-sensitive wild-type strain GJV1 with pTraA and selecting kanamycin-resistant clones. The resulting strain GJV1:pTraA thus carries two truncated copies of traA. Colony-encounter assays involving GJV1, GJV1:pTraA or DK101 were performed on $0.1 \%$ casitone CTT $1.5 \%$ agar.

\section{3 | RESULTS}

\section{1 | TraA diversity and phylogenies among natural isolates}

Eighteen distinct traA alleles were found among the $78 \mathrm{~cm}$-scale Tübingen isolates examined here, and these alleles translated into only fourteen PA14-domain amino acid sequence variants (Figure 2a; Supporting Information Table S1). A rarefaction curve for PA14domain allelic richness demonstrates a rapidly declining, but nonconvergent trajectory of additional diversity with increased sampling effort (Supporting Information Figure S1). The phylogenetic tree topology of $78 \mathrm{~cm}$-scale isolates based on traA (Figure 2a) offers both fine-scale resolution and strong statistical support while closely matching a tree representing much fewer, mainly globally distributed isolates examined by Pathak et al. (2013), which is based on the aligned PA14-region of TraA (Figure 3). It is noticeable that natural isolates are genetically much more diverse at the traA locus compared to more conserved housekeeping loci (MLST), which indicates generally faster rates of sequence evolution in this gene (Figure 4). However, despite this diversity, it is intriguing that almost all of the most prominent TraA subclades present among the global strains are already represented at the smaller $\mathrm{cm}$-scale (Figure $5 \mathrm{a}$ ). This result suggests that there are only a very small number of functionally distinct TraA allotypes worldwide in this species, particularly in comparison with the extremely large number of CMI types previously found among even local populations of relatively low genetic diversity (Kraemer et al., 2016; Vos \& Velicer, 2009).

\section{2 | traA alleles undergo horizontal gene transfer across intraspecific genomic backgrounds}

We analysed the degree to which pairwise genetic relatedness at traA is correlated with overall genomic relatedness as represented by 


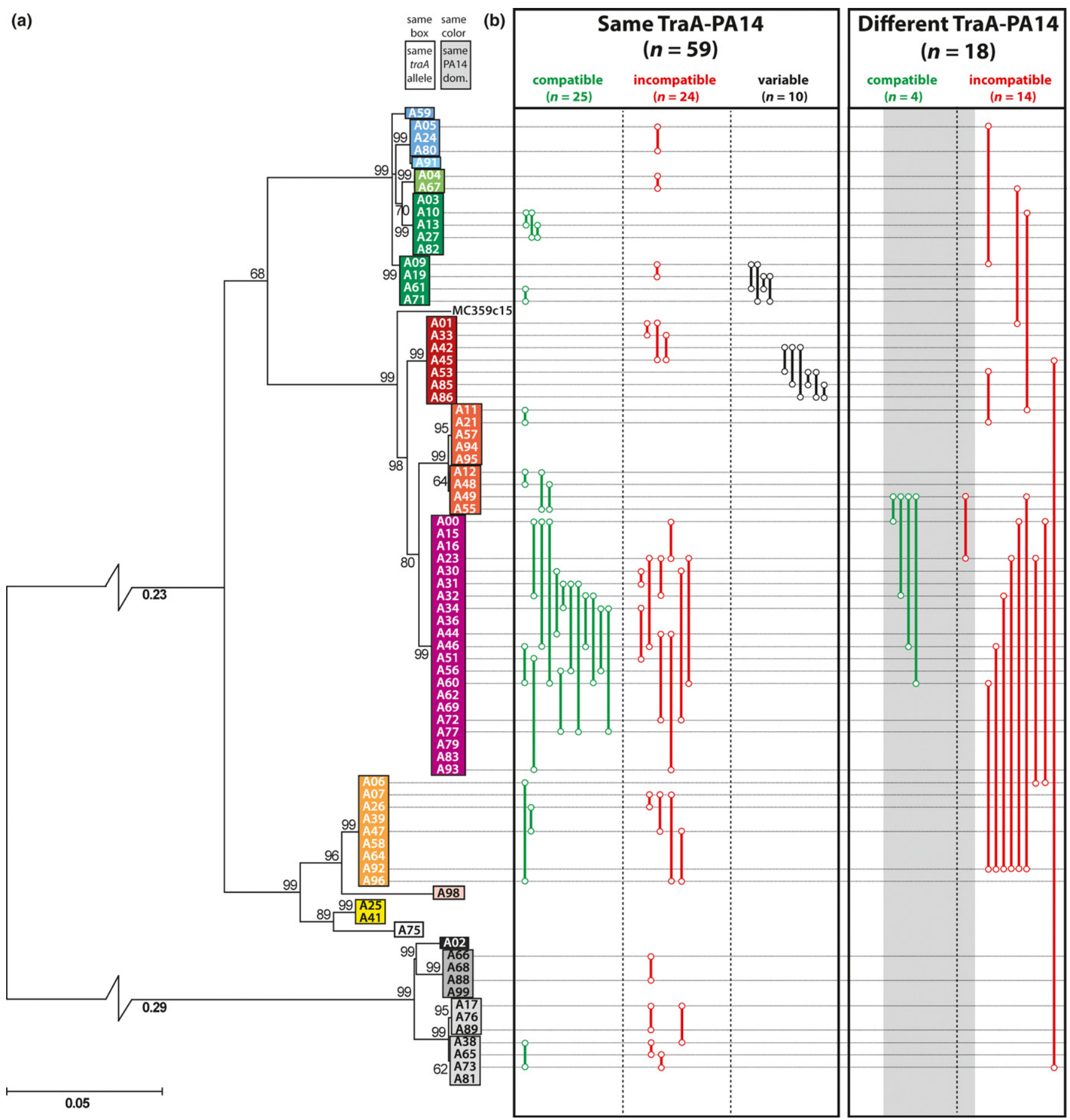

FIGURE 2 Colony-merger social identity is much more diversified than predicted TraA molecular compatibility among local $\mathrm{cm}$-scale isolates of Myxococcus xanthus. (a) Phylogenetic relationships of traA genes among 78 sympatric M. xanthus isolates sampled at the $\mathrm{cm}$-scale and (b) colony-interface phenotypes for 77 isolate pairs are shown. Interaction phenotypes for paired strains sharing the same traA allele are depicted on the left, while those for paired strains with distinct traA alleles are shown on the right. Green, red and black connectors indicate compatible, incompatible and variable colony-interface phenotypes, respectively. The dark grey background shading highlights seven strain pairs with distinct traA nucleotide sequence alleles for which the corresponding TraA proteins may nonetheless be functionally compatible due to phylogenetic proximity. Bootstrap support for each node is indicated, and the very deep split in the tree was broken to allow for better visibility of taxon labels, with respective branch lengths indicated in bold below the branches. Natural isolates contained in the same box share the same traA nucleotide sequence, and different boxes with the same colour share the same PA14-domain at the amino acid sequence level

genetic variation of MLSTs and found that they are significantly but weakly correlated (Pearson's $r=0.32, p<10^{-5}$ according to 10,000 simulations from a Mantel (1967) test). The low magnitude of this correlation suggests that the traA locus often evolves independently of its genetic background. Indeed, a comparison of the traA phylogeny presented here (Figure 2a) with previously published 


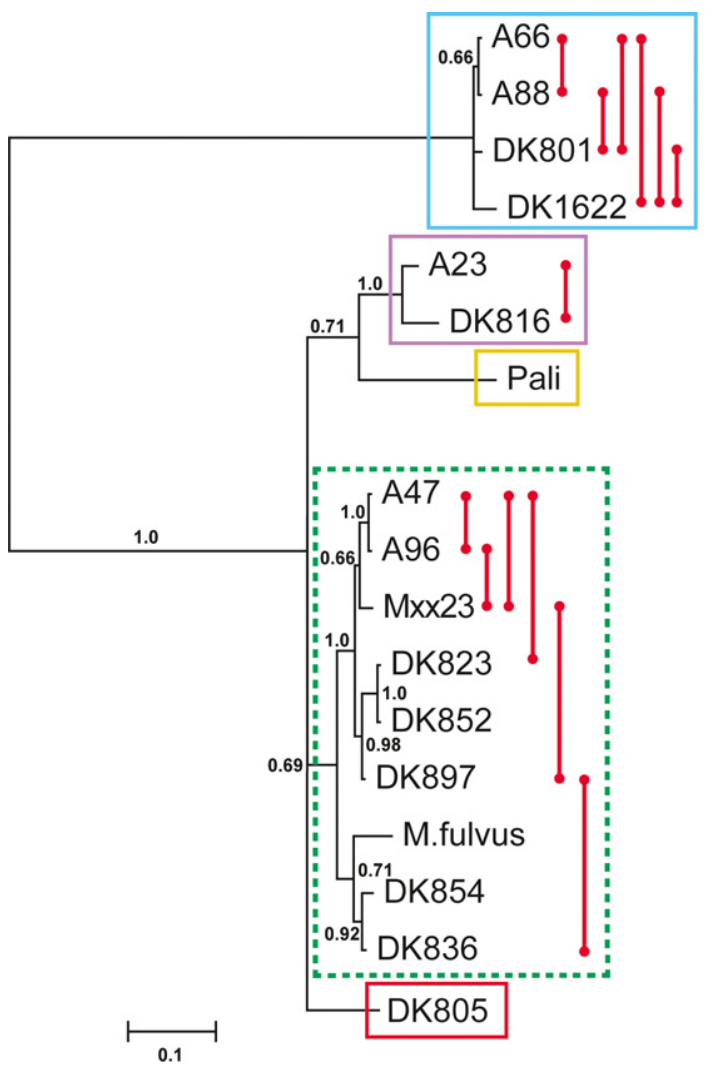

FIGURE 3 TraA-compatible strains pervasively exhibit divergent social identities among global-scale isolates. All of the 13 tested strain pairs predicted by Pathak et al. (2013) to be compatible at TraA for OME generate strong CMIs (red connectors). Coloured boxes depict sets of strains similar at TraA and thus capable of OME during experimentally forced interactions, except the dashed-green box includes both OME-compatible and OME-incompatible strain pairs (Pathak et al., 2013). Strain pairs within the green box designated by the red connectors were predicted by Pathak et al. (2013) to be compatible for OME. The Bayesian phylogeny was inferred based on the amino acid alignment of the PA14-portion of TraA and using the same settings as Pathak et al. (2013). Statistical support values (posterior probabilities) are highlighted on the tree. Strains beginning with " $A$ " are from the focal $\mathrm{cm}$-scale Tübingen population examined in Figure 2, DK1622 is a standard laboratory reference strain, and the remaining strains are other natural isolates from globally distributed origins

phylogenies based on multiple loci (that are more likely to reflect average patterns of genomic relatedness; Vos \& Velicer, 2006, 2009) indicated that traA alleles frequently transfer across highly divergent M. xanthus genomes. To demonstrate this explicitly, we reconstructed an MLST phylogeny based on four genes (csgA, fibA, sgIK and $r p o D)$ for a majority (11/17) of strains examined by Pathak et al. (2013) — thus, also including global isolates — and compared its structure to a traA phylogeny for the same strains. This analysis reveals several striking examples of phylogenetic incongruity (Figure 4). For example, the MLSTs of A23, A47 and A96 are identical and hence form a monophyletic group in the MLST tree, but A23 branches deeply away from $A 47$ and $A 96$ on the traA tree (with the latter two being identical at traA). Likewise, the traA sequence of DK801 is similar to derived sequences of DK1622, A66 and A88, but branches deeply away from all of these other strains on the MLST phylogeny. This qualitative result was further bolstered by quantitative analyses by binning each traA allele into its respective MLST group for all cmscale isolates (Supporting Information Table S1). In particular, among a total of 14 such groups, MLST clusters 1, 7 and 9 (representing 23/71 binned clones) show especially highly elevated genetic diversities at $\pi \sim 81.99, \sim 155.2$ and $\sim 106.8$, respectively, compared to the weighted average genetic diversity within groups of just $\pi \sim 37.0$. Moreover, the same MLST groups show elevated haplotype diversities $(0.38>h>0.52)$ at traA. This evidence of frequent horizontal transfer of traA alleles across divergent genomic backgrounds indicates that traA alleles in a given genomic background cannot be assumed to have tightly coevolved with the majority of that background.

\section{3 | TraA PA14-domain variants appear to be maintained by balancing selection}

We first determined whether recombination would impact subsequent statistical tests for selection. Consistent with the above analyses, we found strong evidence for the former and identified recombination breakpoints at positions 99 and 463, both situated in the variable PA14-like domain. However, tests for phylogenetic incongruence left and right of the respective recombination breakpoints were clearly insignificant ( $p>0.99$ each), such that any impact of recombination on neutrality tests is negligible. Consequently, we could employ two statistics assessing neutrality at the molecular level, Tajima's $D$ and Fu and Li's F. Both statistics were significantly positive when applied to the entire traA gene (Table 1). Positive values of Tajima's $D$ and Fu \& Li's F statistics are interpretable as due to either a sudden population decline (i.e., population bottlenecks) or the presence of balancing selection (Barreiro \& Quintana-Murci, 2010). Furthermore, variation in the values of these statistics when applied to distinct subregions of traA allows us to distinguish between these hypotheses. In particular, values from both tests are significantly positive only for the PA14-region of traA but not for the cysteine-rich region (Table 1), thus making it unlikely that population decline accounts for our observed pattern. This indicates the operation of balancing selection on the PA14 portion of traA, that is, selection that maintains variation, such as frequencydependent selection, nontransitive fitness relationships and spatiotemporally shifting fitness ranks across heterogeneous environments.

\subsection{TraA-allotype identity does not explain phylogenetic patterns of intercolony kin discrimination}

We examined colony-interface phenotypes for 77 strain pairs that featured 51 of the 78 isolates in the Tübingen $\mathrm{cm}$-scale collection (see Figure 1 for examples). Among these pairs, 59 have the same traA allele (shared by both paired strains) and 18 have distinct alleles 
(a) MLST

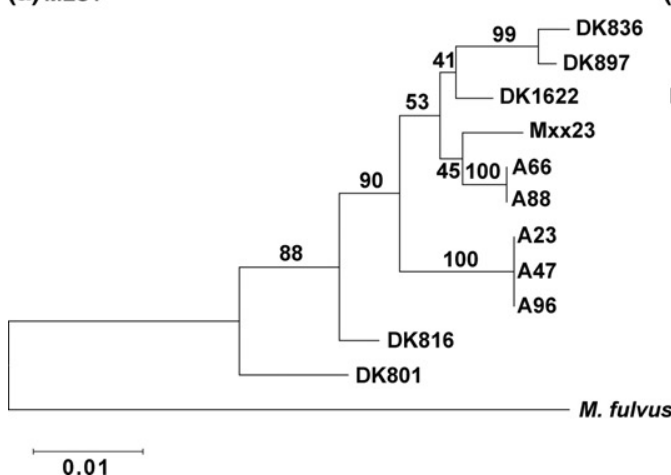

(b) $\operatorname{traA}$

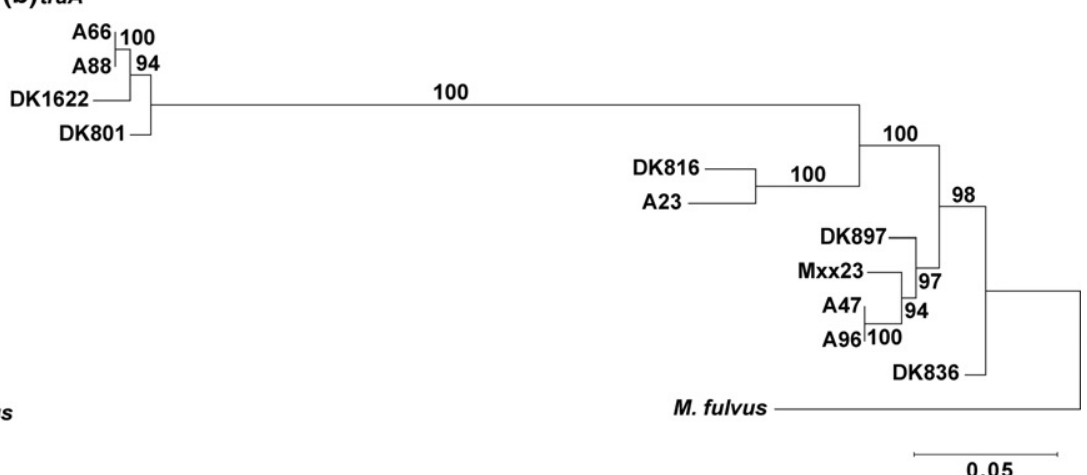

FIGURE 4 Evidence for extensive recombination of the traA gene across genetic backgrounds. The latter was defined by a four-gene multilocus sequence tag (MLST) comprised of csgA, fibA, sglK and rpoD. The data set consists of all global isolates for which both (a) MLST and (b) traA sequence data were available in GenBank. Both phylogenetic trees were inferred using maximum likelihood and rooted using the sister species Myxococcus fulvus. Values on each branch highlight statistical support of nodes after bootstrapping

between pair members (Figure $2 \mathrm{~b}$ ). Also, based on phylogenetic position of the PA14 region amino acid sequence of TraA and the prior functional classifications of TraA variants by Pathak et al. (2013), 66 of these pairs are predicted to be compatible for OME between the pair members while 11 are predicted to be incompatible (Figure 2b). Overall across all 77 strain pairings, 29 pairs were visually compatible for colony merger, whereas 38 pairs exhibited clear and consistent CMls. Ten pairs exhibited visually weaker incompatibilities that varied in strength across replicate experiments (Figure $2 b$ ).

For the 59 strain pairs in which both strains share the same traA allele, if cells in separate colonies of the paired genotypes make physical contact when colonies approach one another, TraA compatibility should allow OME to occur at the colony interface for these pairings. Of those 59 pairs, 25 were found to be fully compatible for colony merger, 24 exhibited clear and consistent CMls and ten exhibited weaker and phenotypically variable CMls (Figure 2b). The high frequency of CMls among these pairs demonstrates that selfidentity for colony merger is often (and perhaps always) determined by genetic factors other than functional dissimilarity at traA among these local isolates (Figure 2b).

Among the $18 \mathrm{~cm}$-scale pairs in which the partners have divergent traA alleles, we nonetheless predict that seven of these pairs should be compatible for OME based on their high degree of similarity between the distinct alleles (grey shaded area in Figure 2b). Among these pairs, four are compatible for colony merger and three exhibit clear CMls. The remaining eleven pairs show strong kin discrimination phenotypes while being highly likely incompatible for OME based on their phylogenetic divergence and functional allotype categories (as summarized in Figure 5a; OME categories and A/P status at the equivalent of DK1622 TraA residue 205, which can determine OME compatibility in some cases (Cao \& Wall, 2017), are listed in Supporting Information Table S2). Overall, these results demonstrate that CMls diversify much more extensively in $M$. xanthus than does TraA functionality. In only this small set of local $\mathrm{cm}$-scale isolates, the diversity of social identity for colony merger (at least 45 CMI allotypes among all 78 strains as determined previously; Vos \&
Velicer, 2009) is more than sixfold greater than predicted the seven predicted functionally distinct TraA allotypes among a phylogenetically broader set of isolates (Figure 3; Pathak et al., 2013).

The high frequency of CMls between closely related local isolates that are identical at traA (Figure 2) suggests that CMls should be even more common among more distantly related strains that are nonetheless compatible at TraA for OME. We examined the colonyinterface phenotypes for 13 pairs of isolates from globally distributed origins studied by Pathak et al. (2013) for which pair members were reported as belonging to the same TraA compatibility type. All 13 pairs (i.e., 100\%) exhibited strong and consistent CMls, strongly suggesting that incompatibility for colony merger is generally not determined by functional dissimilarity at TraA in natural populations (Figure 3).

\section{5 | Disruption of traA does not affect colony- interface phenotypes involving two progenitors of the traA mutant}

Substantial divergence in the PA14 region of the predicted adhesin TraA might, in theory, cause CMls between strains either by (a) preventing $\mathrm{OME}$, or (b) a behavioural response to physical contact between divergent TraA variants independent of OME. Our observation that many pairs of natural isolates with OME-compatible PA14 regions exhibit CMIs (Figure 3 ) strongly suggests that functional divergence at TraA is not the primary cause of CMIs in natural populations.

We did observe that all strain pairs compatible for colony merger are also predicted to be functionally compatible at TraA for OME. This might be taken to suggest that OME is necessary for colony merger in the first place. However, we interpret this result to simply reflect that functional diversification at TraA is much lower than diversification of colony-merger compatibility types (Figures 2 and 3 ), such that if any two strains remain closely related enough across all CMI loci to allow colony merger, it is unlikely they will have functionally diversified at TraA. 
(a)

(b)

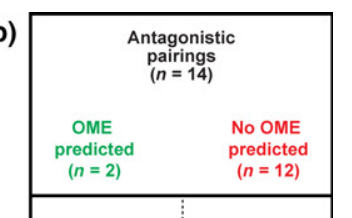

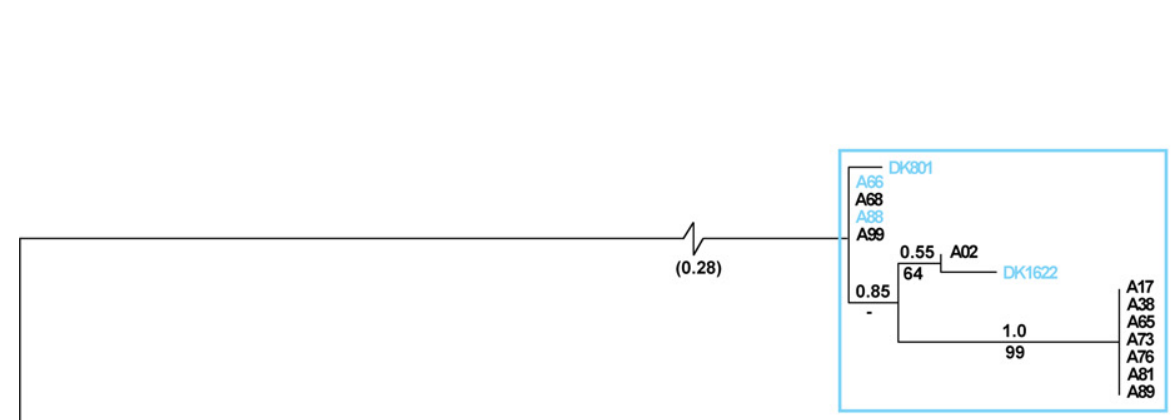

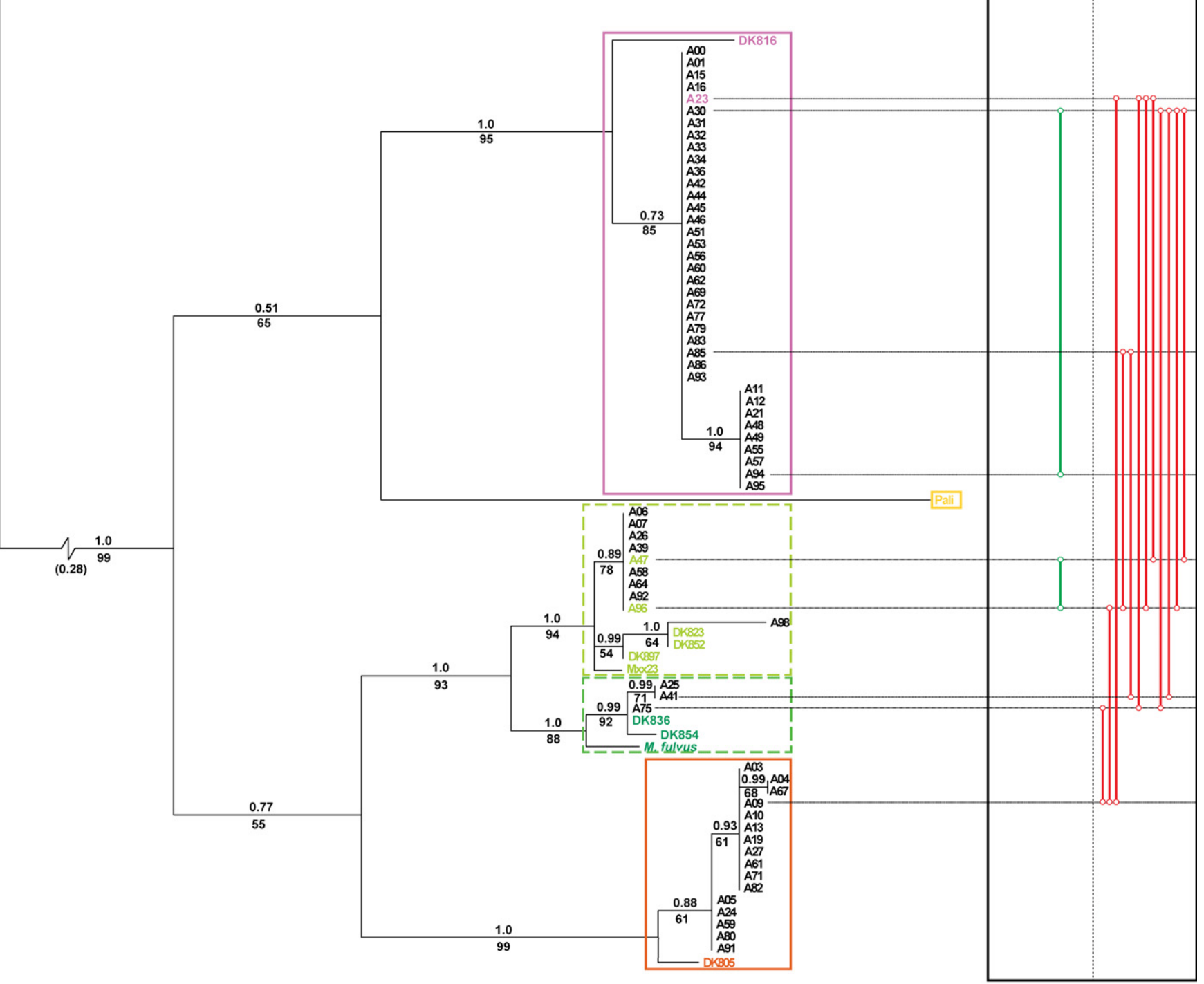

0.1

FIGURE 5 Pairs of natural isolates exhibiting strong interstrain antagonisms are predicted to be largely incompatible for outer membrane exchange (OME). (a) Overall phylogenetic relationships based on amino acid sequences of the TraA-PA14 domain for both the global (Pathak et al., 2013) and the local cm-scale isolates examined here. Coloured rectangles are drawn to encompass all strains predicted by Cao and Wall (2017) to belong to each of several functional TraA categories. Broken branch lengths are indicated in brackets. Values above branches indicate posterior probabilities of Bayesian tree inference, while values below branches depict bootstrap support for the same clades from maximum-likelihood phylogenetic analyses, respectively. (b) Predicted OME compatibility/incompatibility among antagonistic strain pairs ( $n=14$; Rendueles, Amherd et al., 2015) based on phylogenetic proximity of cm-scale to isolates tested for OME and A/P identity at the equivalent of amino acid residue 205 in strain DK1622 (Supporting Information Table S2; Cao \& Wall, 2017; Pathak et al., 2013 ); cf. Figure 3. Green connectors highlight predicted compatibility for OME $(n=2)$, while red connectors depict that OME is improbable $(n=12)$ 
TABLE 1 Evidence for balancing selection among traA alleles is specific to the PA14-domain

\begin{tabular}{lll} 
Domain (traA) & Tajima's D & Fu \& Li's F \\
Whole gene & $1.52^{*}$ & $1.96^{* *}$ \\
\hline PA14-like region & $1.77^{*}$ & $2.33^{* *}$ \\
Cys-rich-region & 1.03 & 1.20 \\
\hline
\end{tabular}

Note. $* p<0.05$ (significance level for Tajima's D); $* * p<0.02$ (significance level for Fu \& Li's F).

We addressed the hypothesis that OME is necessary for colony merger experimentally by disrupting the traA gene in the laboratory reference strain GJV1 to generate two partial copies of the 2,160-nt gene, one lacking the first 68 nucleotides and the other lacking the terminal 1,306 nucleotides. Disruption of traA did not cause CMls between separate colonies of the traA mutant or between the mutant and its parent GJV1 (Supporting Information Figure S2). A similar result published after submission of this study was reported by Vassallo et al. (2017). In particular, disruption of traA did not prevent fruiting bodies from forming on low-nutrient medium along the colony interfaces. In contrast, as a positive control example of $\mathrm{CM}$ formation involving GJV1, fruiting bodies fail to form at the interface of colonies of GJV1 and genetically distinct strain DK101. Taken together, both our comparative analyses of natural isolates and our genetic disruption experiment do not support the hypothesis that interactions between functionally divergent TraA variants contribute to CMls in natural populations.

\subsection{A majority of documented interstrain antagonisms are predicted to be independent of TraA sequence}

Using our phylogenetic data, we tested whether previously documented instances of strong antagonism between paired Tübingen $\mathrm{cm}$-scale isolates occur between strains sharing functionally compatible traA alleles and thus might be mediated by OME (Cao \& Wall, 2017; Pathak et al., 2013). Vos and Velicer (2009) demonstrated strong interstrain antagonisms in 14 pairs of $\mathrm{cm}$-scale isolates when strains were experimentally mixed prior to the onset of multicellular development. Following on these results, Rendueles, Amherd et al. (2015) showed that in most of these pair mixes, the winning strain caused the spore production of the losing strain to decrease by multiple orders of magnitude and these antagonisms were in at least some cases lethal, indicating the involvement of toxins, and in some cases also appeared to be contact dependent.

Among the 14 antagonistic pairings examined by Rendueles, Amherd et al. (2015) involving nine $\mathrm{cm}$-scale isolates, all but two (A47-A96 and A30-A94) involve paired strains we predict to be incapable of OME due to incompatible PA14-regions of TraA (Figure $5 \mathrm{~b}$ ). These predictions are based on the phylogenetic positions of paired strains relative to other strains previously assigned to functional TraA-PA14 categories (Figure 5a) by Pathak et al. (2013) and are further strengthened by the fact all clones within each coloured box of Figure 5a share the same amino acid state (amino acids $A$ or $P$ at the position equivalent to position 205 in reference strain DK1622) that was recently found to determine OME compatibility for some strain combinations (Cao \& Wall, 2017). Given that these strain pairs were selected based on previous evidence of strong antagonism relative to other strain pairs that exhibited less antagonism (Vos \& Velicer, 2009), these results strongly suggest that the majority of latent interstrain antagonisms among strains that might interact within local populations are not mediated by OME.

\section{DISCUSSION}

Kin discrimination can profoundly shape patterns of interaction, cooperation and conflict among microbes and the molecular causes of kin discrimination phenotypes remain of considerable interest (Gibbs, Urbanowski, \& Greenberg, 2008; Kraemer et al., 2016; Lyons, Kraigher, Stefanic, Mandic-Mulec, \& Kolter, 2016; Rendueles, Amherd et al., 2015; Rendueles, Zee et al., 2015; Stefanic et al., 2015; Strassmann et al., 2011). In M. xanthus, one cell-surface protein that has been proposed to influence patterns of social interaction in natural populations of the myxobacteria is TraA, which mediates OME, a category of cell-cell interaction of unknown selective benefit. Analysing a focal natural population of M. xanthus sampled at the centimetre scale, we find that most (or all) of several TraA-allotype classes present among samples from globally distributed origins (Pathak et al., 2013) appear to coexist also in our focal $\mathrm{cm}$-scale population. While relatedness at traA correlates weakly with an MLST-based measure of overall genomic relatedness, there is clear evidence that traA alleles frequently transfer horizontally across genomic backgrounds.

Further, we asked whether interaction between distinct functional TraA allotypes at colony interfaces might contribute to CMIs among natural isolates. Although all $\mathrm{cm}$-scale strain pairs predicted to be nonfunctional for OME due to different TraA-PA14 regions exhibit CMIs (Figure 2), among strain pairs likely to be functional for OME due to similarity at traA, approximately half also exhibit CMls. Moreover, all examined pairs of more globally distributed isolates predicted to be compatible for OME also exhibited CMIs. The pervasive evolution of diverse CMIs within predicted TraA-allotype categories indicates that, as in the experimental populations of Rendueles, Zee et al. (2015), diversification of CMI self-identity in natural populations of $M$. xanthus is not driven by diversification of TraA functional identity, which is clearly much lower than CMI diversification. Natural M. xanthus CMls have been predicted to involve many loci (Rendueles, Zee et al., 2015; Wielgoss et al., 2016), as is the case for CMls among natural isolates of B. subtilis (Lyons et al., 2016).

Intriguingly, Dey et al. (2016) showed that M. xanthus laboratory reference strains harbouring three copies of the prophage $M x$ alpha could kill both closely related and unrelated Myxococcus strains that lacked one or more of these copies. This form of antagonism was mediated by TraAB-dependent OME and it was proposed that killing 
and immunity was based on a toxin/antitoxin system encoded in the prophage sequences. According to this hypothesis, carriers of the same traA allele may engage in OME upon close cell-cell contact and transfer one or several toxins. Recipient cells carrying a specific importer, OmrA, but lacking the necessary antitoxin will be killed, as they lack immunity (Dey \& Wall, 2014). The same prophage sequences were found to be localized in a narrow genomic region of the $M$. xanthus genome that is highly variable in gene content within a 22-isolate subset of the $78 \mathrm{~cm}$-scale natural M.xanthus isolates examined here (Wielgoss et al., 2016). Consequently, Wielgoss et al. (2016) suggested that gene-content variation within this region, which includes a homolog to the Burkholderia contact-dependent growth inhibition gene cdiA (aka $b c p A$ ), might be responsible for some of the interstrain antagonisms among Tübingen $\mathrm{cm}$-scale natural isolates. More recently, Vassallo et al. (2017) showed that such OME-mediated toxin delivery has the potential to generate CMls between strains that differ in their toxin/antitoxin gene content but are otherwise isogenic.

If OME-mediated antagonisms between natural strains are common, this might impose positive selection for OME incompatibilities, which could benefit genotypes sensitive to an OME-mediated toxin produced by another strain with which it interacts. In principle, such selection could drive TraA functional diversification (as hypothesized by Vassallo et al., 2017), but the very small number of TraA functional classes found to date in M. xanthus indicates that even if there is such direct selection for actualized TraA incompatibilities (i.e., incompatibility between TraA variants that actually make contact in nature), its power to drive diversification appears to be very limited. Here, we have predicted that most strain pairs known to exhibit strong interstrain antagonisms are predicted to be incompatible for OME based on the phylogenetic position of their TraA-PA14 domain alleles (Figure 5). Thus, data from this focal population (including five isolates used in the OME analysis by Pathak et al. (2013)) suggest that most interstrain antagonisms in $M$. xanthus are unrelated to OME. Collectively, our results do not support the hypothesis that TraA functional identity plays a major causal role in determining documented patterns of kin discrimination in natural populations of M. xanthus, either by TraA incompatibilities causing CMIs or by OMEmediated toxin transfer between TraA-compatible strains causing interstrain antagonisms. Whether OME-mediated toxin transfer contributes to some CMls between natural isolates remains to be tested.

We also found evidence that distinct alleles for the PA14-encoding segment of traA appear to be under balancing selection. Similar evidence for balancing selection among the same $78 \mathrm{~cm}$-scale isolates as examined here has been found for the type IV-pilus gene pilA involved in M. xanthus social motility (Vos \& Velicer, 2006). One form of balancing selection relevant to prokaryotes is frequencydependent selection, but from current understanding of the mechanistic roles that pilA and traA may play in $M$. xanthus biology, there is no obvious rationale to suggest that the fitness effects of alleles at these loci should correlate inversely with their frequency in natural populations due to social interactions between distinct allelic types. Alternatively, balancing selection in prokaryotes can also be generated when alleles vary in their fitness effects across spatially and temporally heterogeneous soil environments and this hypothesis may explain why multiple PilA and TraA variants appear to be maintained by selection in our focal $\mathrm{cm}$-scale population.

Importantly, the signal of balancing selection among traA alleles does not imply that TraA functional incompatibilities per se are favoured by selection. Latent TraA incompatibilities (i.e., incompatibilities not necessarily manifested in real cell-cell interactions) may have evolved indirectly as by-products of diversification that was caused by something other than selection acting directly on interactions between TraA variants. In this view, selection would act primarily on any functional effects of new mutations in traA, whether with respect to OME between cells identical for the new traA allele or with respect to some advantageous function other than OME that TraA may mediate, such as cell-cell adhesion, communication or metabolism (Rigden et al., 2004; Taylor \& Drickamer, 2014; Zupancic et al., 2008). Those mutations having a negative effect on a beneficial function of TraA (whatever it may be) would be lost by selection whereas new TraA variants that do not reduce or even enhance fitness during interactions between cells bearing the same TraA type would not be purged by negative selection. The finding that most strong antagonisms in our focal $\mathrm{cm}$-scale population appear to be independent of OME (Figure 5) is consistent with the hypothesis that TraA incompatibilities evolve predominantly as indirect by-products of selection on something other than those incompatibilities per se.

The suites of cell-surface and diffusible molecules produced by the myxobacteria that have the potential to influence patterns of social interaction are highly complex (Curtis, Taylor, Welch, \& Shimkets, 2007) and thus potentiate myriad routes to social diversification (Rendueles, Zee et al., 2015). Which functional differences in social molecules play primary causal roles in shaping patterns of intergenotype social interactions in natural populations and which differences remain largely latent due to more functionally primary differences preventing their manifestation is an important theme for future investigations (Velicer, Mendes-Soares, \& Wielgoss, 2014). For example, actualized primary incompatibilities between genotypes may prevent some colonies from merging and coaggregating into common fruiting bodies, thereby preventing any latent developmental incompatibilities between the genotypes from ever being manifested. To date, diversification of TraA functionality appears to be very limited, especially relative to diversification at a demonstrable form of social identity manifested during M. xanthus-generated interactions (i.e., motility-driven colony encounters). Thus, TraA incompatibilities may remain predominantly latent rather than actualized in natural populations. Whether TraA functional incompatibilities play a substantial role in determining which myxobacterial genotypes cooperate during motility, predation or development in natural populations remains unclear.

\section{ACKNOWLEDGEMENTS}

We thank Guillaume Achaz and the three independent reviewers for helpful comments. This work was supported by a EU Marie Curie 
PEOPLE Postdoctoral Fellowship for Career Development grant (FP7-PEOPLE-2012-IEF-331824) to S.W. and an EMBO Long-Term Fellowship to O.R.

\section{DATA ACCESSIBILITY}

Individual data sets for statistical analyses are provided at the Dryad Digital Repository: https://doi.org/10.5061/dryad.m5f7b44. Correspondence and request for materials should be addressed to G.J.V (gregory.velicer@env.ethz.ch) or S.W. (sebastien.wielgoss@env.ethz.ch).

\section{COMPETING INTERESTS}

The authors declare no conflict of interest.

\section{AUTHOR CONTRIBUTIONS}

S.W. and O.R. conducted sequencing analyses, F.F. and Y.-T.N.Y. conducted experiments, S.W. and O.R. performed phylogenetic and statistical analyses, S.W. and G.J.V. wrote the original manuscript, and all authors participated in manuscript revision.

\section{ORCID}

Sébastien Wielgoss (iD http://orcid.org/0000-0002-0127-3380

\section{REFERENCES}

Barreiro, L. B., \& Quintana-Murci, L. (2010). From evolutionary genetics to human immunology: How selection shapes host defence genes. Nature Reviews Genetics, 11, 17-30. https://doi.org/10.1038/ nrg2698

Bretl, D. J., \& Kirby, J. R. (2016). Molecular mechanisms of signaling in Myxococcus xanthus development. Journal of Molecular Biology, 428, 3805-3830. https://doi.org/10.1016/j.jmb.2016.07.008

Bretscher, A. P., \& Kaiser, D. (1978). Nutrition of Myxococcus xanthus, a fruiting myxobacterium. Journal of Bacteriology, 133, 763-768.

Budding, A. E., Ingham, C. J., Bitter, W., Vandenbroucke-Grauls, C. M., \& Schneeberger, P. M. (2009). The Dienes phenomenon: Competition and territoriality in swarming Proteus mirabilis. Journal of Bacteriology, 191, 3892-3900. https://doi.org/10.1128/JB.00975-08

Cao, P., Dey, A., Vassallo, C. N., \& Wall, D. (2015). How myxobacteria cooperate. Journal of Molecular Biology, 427, 3709-3721. https://doi. org/10.1016/j.jmb.2015.07.022

Cao, P., \& Wall, D. (2017). Self-identity reprogrammed by a single residue switch in a cell surface receptor of a social bacterium. Proceedings of the National Academy of Sciences of the United States of America, 114, 3732-3737. https://doi.org/10.1073/pnas.1700315114

Chen, H. W., Kuspa, A., Keseler, I. M., \& Shimkets, L. J. (1991). Physical map of the Myxococcus xanthus chromosome. Journal of Bacteriology, 173, 2109-2115. https://doi.org/10.1128/jb.173.6.2109-2115.1991

Chessel, D., Dufour, A. B., \& Thioulouse, J. (2004). The ade4 package - I: One-table methods. $R$ News, 4, 5-10.

Collier, R. J., \& Young, J. A. T. (2003). Anthrax toxin. Annual Review of Cell and Developmental Biology, 19, 45-70. https://doi.org/10.1146/an nurev.cellbio.19.111301.140655

Curtis, P. D., Taylor, R. G., Welch, R. D., \& Shimkets, L. J. (2007). Spatial organization of Myxococcus xanthus during fruiting body formation.
Journal of Bacteriology, 189, 9126-9130. https://doi.org/10.1128/JB. 01008-07

Darriba, D., Taboada, G. L., Doallo, R., \& Posada, D. (2011). ProtTest 3: Fast selection of best-fit models of protein evolution. Bioinformatics, 27, 1164-1165. https://doi.org/10.1093/bioinformatics/btr088

Delport, W., Poon, A. F. Y., Frost, S. D. W., \& Pond, S. L. K. (2010). Datamonkey 2010: A suite of phylogenetic analysis tools for evolutionary biology. Bioinformatics, 26, 2455-2457. https://doi.org/10.1093/bioin formatics/btq429

Dey, A., Vassallo, C. N., Conklin, A. C., Pathak, D. T., Troselj, V., \& Wall, D. (2016). Sibling rivalry in Myxococcus xanthus is mediated by kin recognition and a polyploid prophage. Journal of Bacteriology, 198, 994-1004. https://doi.org/10.1128/JB.00964-15

Dey, A., \& Wall, D. (2014). A genetic screen in Myxococcus xanthus identifies mutants that uncouple outer membrane exchange from a downstream cellular response. Journal of Bacteriology, 196, 4324-4332. https://doi.org/10.1128/JB.02217-14

Dienes, L. (1946). Reproductive processes in Proteus cultures. Proceedings of the Society for Experimental Biology and Medicine, 63, 265-270. https://doi.org/10.3181/00379727-63-15570

Ducret, A, Fleuchot, B, Bergam, P, \& Mignot, T (2013). Direct live imaging of cell-cell protein transfer by transient outer membrane fusion in Myxococcus xanthus. eLife, 2, e00868.

Fiegna, F., \& Velicer, G. J. (2005). Exploitative and hierarchical antagonism in a cooperative bacterium. Plos Biology, 3, 1980-1987.

Fu, Y. X., \& Li, W. H. (1993). Statistical tests of neutrality of mutations. Genetics, 133, 693-709.

Gelman, A., \& Rubin, D. B. (1992). Inference from iterative simulation using multiple sequences. Statistical Science, 7, 457-511. https://doi. org/10.1214/ss/1177011136

Gibbs, K. A., Urbanowski, M. L., \& Greenberg, E. P. (2008). Genetic determinants of self identity and social recognition in bacteria. Science, 321, 256-259. https://doi.org/10.1126/science.1160033

Goossens, K., \& Willaert, R. (2010). Flocculation protein structure and cellcell adhesion mechanism in Saccharomyces cerevisiae. Biotechnology Letters, 32, 1571-1585. https://doi.org/10.1007/s10529-010-0352-3

Hodgkin, J., \& Kaiser, D. (1979). Genetics of gliding motility in Myxococcus xanthus (Myxobacterales) - Genes-controlling movement of single cells. Molecular and General Genetics, 171, 167-176. https://doi.org/ 10.1007/BF00270003

Kaiser, D., \& Welch, R. (2004). Dynamics of fruiting body morphogenesis. Journal of Bacteriology, 186, 919-927. https://doi.org/10.1128/JB. 186.4.919-927.2004

Kraemer, S. A., \& Velicer, G. J. (2011). Endemic social diversity within natural kin groups of a cooperative bacterium. Proceedings of the National Academy of Sciences of the United States of America, 108, 10823-10830. https://doi.org/10.1073/pnas.1100307108

Kraemer, S. A., Wielgoss, S., Fiegna, F., \& Velicer, G. J. (2016). The biogeography of kin discrimination across microbial neighbourhoods. Molecular Ecology, 25, 4875-4888. https://doi.org/10.1111/mec.13803

Lyons, N. A., Kraigher, B., Stefanic, P., Mandic-Mulec, I., \& Kolter, R. (2016). A combinatorial kin discrimination system in Bacillus subtilis. Current Biology, 26, 733-742. https://doi.org/10.1016/j.cub.2016.01.032

Mantel, N. (1967). The detection of disease clustering and a generalized regression approach. Cancer Research, 27, 209-220.

Nudleman, E., Wall, D., \& Kaiser, D. (2005). Cell-to-cell transfer of bacterial outer membrane lipoproteins. Science, 309, 125-127. https://doi. org/10.1126/science. 1112440

Oohata, A. A., Nakagawa, M., Tasaka, M., \& Fujii, S. (1997). A novel prespore-cell-inducing factor in Dictyostelium discoideum induces cell division of prespore cells. Development, 124, 2781-2787.

Pathak, D. T., Wei, X., Bucuvalas, A., Haft, D. H., Gerloff, D. L., \& Wall, D. (2012). Cell contact-dependent outer membrane exchange in myxobacteria: Genetic determinants and mechanism. PLoS Genetics, 8, e1002626. https://doi.org/10.1371/journal.pgen.1002626 
Pathak, D. T., Wei, X., Dey, A., \& Wall, D. (2013). Molecular recognition by a polymorphic cell surface receptor governs cooperative behaviors in bacteria. PLoS Genetics, 9, e1003891. https://doi.org/10.1371/jour nal.pgen.1003891

Pond, S. L. K., \& Frost, S. D. W. (2005). Datamonkey: Rapid detection of selective pressure on individual sites of codon alignments. Bioinformatics, 21, 2531-2533. https://doi.org/10.1093/bioinformatics/bti320

Pond, S. L. K., Frost, S. D. W., \& Muse, S. V. (2005). HyPhy: Hypothesis testing using phylogenies. Bioinformatics, 21, 676-679. https://doi. org/10.1093/bioinformatics/bti079

Pond, S. L. K., Posada, D., Gravenor, M. B., Woelk, C. H., \& Frost, S. D. W. (2006). GARD: A genetic algorithm for recombination detection. Bioinformatics, 22, 3096-3098. https://doi.org/10.1093/bioinformatics/btl474

Rajagopalan, R., Wielgoss, S., Lippert, G., Velicer, G. J., \& Kroos, L. (2015). devl is an evolutionarily young negative regulator of Myxococcus xanthus development. Journal of Bacteriology, 197, 1249-1262. https://doi.org/10.1128/JB.02542-14

Ramirez-Soriano, A., Ramos-Onsins, S. E., Rozas, J., Calafell, F., \& Navarro, A. (2008). Statistical power analysis of neutrality tests under demographic expansions, contractions and bottlenecks with recombination. Genetics, 179, 555-567. https://doi.org/10.1534/genetics. 107.083006

Rendueles, O., Amherd, M., \& Velicer, G. J. (2015). Positively frequencydependent interference competition maintains diversity and pervades a natural population of cooperative microbes. Current Biology, 25, 1673-1681. https://doi.org/10.1016/j.cub.2015.04.057

Rendueles, O., Zee, P. C., Dinkelacker, I., Amherd, M., Wielgoss, S., \& Velicer, G. J. (2015). Rapid and widespread de novo evolution of kin discrimination. Proceedings of the National Academy of Sciences of the United States of America, 112, 9076-9081. https://doi.org/10.1073/ pnas.1502251112

Rigden, D. J., Mello, L. V., \& Galperin, M. Y. (2004). The PA14 domain, a conserved all-beta domain in bacterial toxins, enzymes, adhesins and signaling molecules. Trends in Biochemical Sciences, 29, 335-339. https://doi.org/10.1016/j.tibs.2004.05.002

Ronquist, F., Teslenko, M., van der Mark, P., Ayres, D. L., Darling, A., Höhna, S., ... Huelsenbeck, J. P. (2012). MrBayes 3.2: Efficient Bayesian phylogenetic inference and model choice across a large model space. Systematic Biology, 61, 539-542. https://doi.org/10.1093/sysb io/sys029

Schierup, M. H., \& Hein, J. (2000). Consequences of recombination on traditional phylogenetic analysis. Genetics, 156, 879-891.

Stefanic, P., Kraigher, B., Lyons, N. A., Kolter, R., \& Mandic-Mulec, I. (2015). Kin discrimination between sympatric Bacillus subtilis isolates. Proceedings of the National Academy of Sciences of the United States of America, 112, 14042-14047. https://doi.org/10.1073/pnas.1512671112

Strassmann, J. E., Gilbert, O. M., \& Queller, D. C. (2011). Kin discrimination and cooperation in microbes. Annual Review of Microbiology, 65, 349-367. https://doi.org/10.1146/annurev.micro.112408.134109

Tajima, F. (1989). Statistical method for testing the neutral mutation hypothesis by DNA polymorphism. Genetics, 123, 585-595.

Tamura, K., Stecher, G., Peterson, D., Filipski, A., \& Kumar, S. (2013). MEGA6: Molecular evolutionary genetics analysis version 6.0. Molecular Biology and Evolution, 30, 2725-2729. https://doi.org/10.1093/molbe $\mathrm{v} / \mathrm{mst} 197$

Taylor, M. E., \& Drickamer, K. (2014). Convergent and divergent mechanisms of sugar recognition across kingdoms. Current Opinion in Structural Biology, 28, 14-22. https://doi.org/10.1016/j.sbi.2014.07.003

Vassallo, C. N., Cao, P., Conklin, A., Finkelstein, H., Hayes, C. S., \& Wall, D. (2017). Infectious polymorphic toxins delivered by outer membrane exchange discriminate kin in myxobacteria. Elife, 6, e29397. https://doi.org/10.7554/eLife. 29397

Vassallo, C., Pathak, D. T., Cao, P., Zuckerman, D. M., Hoiczyk, E., \& Wall, D. (2015). Cell rejuvenation and social behaviors promoted by LPS exchange in myxobacteria. Proceedings of the National Academy of
Sciences of the United States of America, 112, E2939-E2946. https://d oi.org/10.1073/pnas.1503553112

Vassallo, C. N., \& Wall, D. (2016). Tissue repair in myxobacteria: A cooperative strategy to heal cellular damage. BioEssays, 38, 306-315. https://doi.org/10.1002/bies.201500132

Velicer, G. J., Mendes-Soares, H., \& Wielgoss, S. (2014). Whence comes social diversity? Ecological and evolutionary analysis of the myxobacteria. In Z. Yang \& P. I. Higgs (Eds.), Myxobacteria: Genomics, cellular and molecular biology (pp. 1-28). Norfolk, UK: Caister Academic Press.

Velicer, G. J., Raddatz, G., Keller, H., Deiss, S., Lanz, C., Dinkelacker, I., \& Schuster, S. C. (2006). Comprehensive mutation identification in an evolved bacterial cooperator and its cheating ancestor. Proceedings of the National Academy of Sciences of the United States of America, 103, 8107-8112. https://doi.org/10.1073/pnas.0510740103

Velicer, G. J., \& Vos, M. (2009). Sociobiology of the myxobacteria. Annual Review of Microbiology, 63, 599-623. https://doi.org/10.1146/annure v.micro.091208.073158

Vos, M., \& Velicer, G. J. (2006). Genetic population structure of the soil bacterium Myxococcus xanthus at the centimeter scale. Applied and Environmental Microbiology, 72, 3615-3625. https://doi.org/10.1128/ AEM.72.5.3615-3625.2006

Vos, M., \& Velicer, G. J. (2008). Isolation by distance in the spore-forming soil bacterium Myxococcus xanthus. Current Biology, 18, 386-391. https://doi.org/10.1016/j.cub.2008.02.050

Vos, M., \& Velicer, G. J. (2009). Social conflict in centimeter and globalscale populations of the bacterium Myxococcus xanthus. Current Biology, 19, 1763-1767. https://doi.org/10.1016/j.cub.2009.08.061

Wall, D. (2014). Molecular recognition in myxobacterial outer membrane exchange: Functional, social and evolutionary implications. Molecular Microbiology, 91, 209-220. https://doi.org/10.1111/mmi.12450

Wall, D. (2016). Kin recognition in bacteria. Annual Review of Microbiology, 70, 143-160. https://doi.org/10.1146/annurev-micro-102215-095325

Wei, X., Pathak, D. T., \& Wall, D. (2011). Heterologous protein transfer within structured myxobacteria biofilms. Molecular Microbiology, 81, 315-326. https://doi.org/10.1111/j.1365-2958.2011.07710.x

Wielgoss, S., Didelot, X., Chaudhuri, R. R., Liu, X., Weedall, G. D., Velicer, G. J., \& Vos, M. (2016). A barrier to homologous recombination between sympatric strains of the cooperative soil bacterium Myxococcus xanthus. The ISME Journal, 10, 2468-2477. https://doi.org/10. 1038/ismej.2016.34

Wu, S. S., \& Kaiser, D. (1995). Genetic and functional evidence that Type IV pili are required for social gliding motility in Myxococcus xanthus. Molecular Microbiology, 18, 547-558. https://doi.org/10.1111/j.13652958.1995.mmi_18030547.x

Zupancic, M. L., Frieman, M., Smith, D., Alvarez, R. A., Cummings, R. D., \& Cormack, B. P. (2008). Glycan microarray analysis of Candida glabrata adhesin ligand specificity. Molecular Microbiology, 68, 547-559. https://doi.org/10.1111/j.1365-2958.2008.06184.x

\section{SUPPORTING INFORMATION}

Additional supporting information may be found online in the Supporting Information section at the end of the article.

How to cite this article: Wielgoss S, Fiegna F, Rendueles $O$, Yu Y-TN, Velicer GJ. Kin discrimination and outer membrane exchange in Myxococcus xanthus: A comparative analysis among natural isolates. Mol Ecol. 2018;00:1-13. https://doi.org/10.1111/mec.14773 